\title{
New Insight into the Treatment of Primary Myelofibrosis
}

\author{
Nahla AM Hamed* \\ Professor of Hematology, Hematology Department, Alexandria University, Egypt
}

Submission: December 03, 2018; Published: December 11, 2018

"Correspondence Address: Nahla AM Hamed, Professor of Hematology, Hematology Department, Alexandria University, Egypt

\begin{abstract}
PMF belongs to the category of myeloproliferative neoplasm. It has a chronic disabling course and shortened survival. The prognostic scores IPSS, DIPSS and DIPSS-plus, do not perform as well for post-ET or post-PV MF patients. Three new prognostic systems have been recently introduced: GIPSS based exclusively on mutations and karyotype, MIPSS70 is best utilized in the absence of cytogenetic information but presence of molecular information and MIPSS70 + version 2.0 utilizes both genetic and clinical risk factors. These scores are used to aid decisions regarding eligibility for stem cell transplant.
\end{abstract}

Abbreviation: PMF: Primary Myelofibrosis; EMH: Extramedullary Hematopoiesis; HMR: High Molecular Risk; IPSS: International Prognostic Scoring System; DIPSS: dynamic IPSS; PV: Polycythemia Vera; ET: Essential Thrombocythemia; GIPSS: Genetically-inspired Prognostic Scoring System; MIPSS70: Mutation and karyotype-enhanced International Prognostic Scoring System for Transplant-age Patients; MIPSS70+ Version 2.0: The karyotype-Enhanced MPPSS70.

\section{Introduction}

Myelofibrosis may present as a primary disorder (PMF) or evolve from polycythemia vera (PV) or essential thrombocythemia (ET) to post-PV or post-ET MF [1]. PMF is a hematopoietic stemcell-derived clonal myeloproliferation that belongs to the category of myeloproliferative neoplasm [2]. It has an incidence of about 0.58 new cases per 100.000 person-years, and a prevalence of 6 per 100.000 person-years. Median age at diagnosis is 67 years, with no significant difference in sex distribution [1]. Aberrant cytokine production by clonal cells and host immune reaction are assumed to contribute to PMF associated bone marrow stromal changes [3] (including reticulin fibrosis) [1], ineffective erythropoiesis, EMH, cachexia and constitutional symptoms [3]. Clinical manifestations in PMF include severe anemia, marked hepatosplenomegaly, constitutional symptoms (e.g., fatigue, night sweats, fever), cachexia, bone pain, splenic infarct, pruritus, thrombosis and bleeding. Ineffective erythropoiesis and EMH are the main causes of anemia and organomegaly, respectively [3].

Diagnosis of MF is currently based on the World Health Organization 2016 criteria, which include bone marrow morphology and presence of the driver mutations JAK2, CALR or MPL in $\sim 90 \%$ (50\% to $60 \%, 15 \%-20 \%$ and $5 \%-10 \%$ respectively) of the patients. Their presence is supportive but not essential for diagnosis. It is generally believed that driver mutations are essential for the MPN phenotype [4]. About 10\% of patients do not develop any known mutation and are considered to have 'triple-negative' MF [1]. In addition to driver mutations, $80 \%$ of PMF patients harbor other DNA variants in myeloid genes, including ASXL1, TET2, EZH2, SRSF2, DNMT3A, U2AF1, and IDH1/IDH2, often in multiple combinations [2]. These mutations might contribute to disease progression and leukemic transformation [4]. ASXL1, SRSF2, EZH2, and IDH1/IDH2 mutations are considered high-molecular risk (HMR) mutations; their prognostic relevance is further amplified by their number in an individual patient [2]. Patients with mutations in $\leq 2$ non-canonical genes had 9-fold higher odds of spleen response to ruxolitinib compared to those with $\geq 3$ mutations in such genes [5].

\section{Prognosis}

PMF has a chronic disabling course [1] and shortened survival [4]. Disease complications include symptomatic portal hypertension that might lead to variceal bleeding or ascites and non-hepatosplenic EMH that might lead to cord compression, ascites, pleural effusion, pulmonary hypertension or diffuse extremity pain. Causes of death include leukemic progression in approximately $20 \%$ of patients, consequences of cytopenias including infection or bleeding and comorbid conditions including cardiovascular events [3].

\section{Risk Stratification}

The prognostic score IPSS, is applicable at diagnosis; DIPSS and DIPSS-plus, can be applied at any time during follow up. These 3 prognostic scoring systems refer to age, constitutional symptoms, anemia, white blood cell counts, and percentage of peripheral blood blasts. DIPSS-plus incorporates 3 additional independent risk factors: red blood cell transfusion requirement, platelet counts $<100 \times 10^{9} / \mathrm{L}$, and an unfavorable karyotype [1]. The prognostic scores: IPSS, DIPSS, and DIPSS-plus do not 


\section{Cancer Therapy \& Oncology International Journal}

perform as well for post-ET or post-PV MF patients [6]. Three new prognostic systems have been recently introduced: GIPSS which is based exclusively on mutations and karyotype [4], MIPSS70 is best utilized in the absence of cytogenetic information but presence of molecular information [7] and MIPSS70+ version 2.0 which utilizes both genetic and clinical risk factors [4]. HMR, or cytogenetic abnormalities, are used to refine prognosis and to aid decisions regarding eligibility for stem cell transplant [6]. GIPSS was also shown to predict leukemic transformation [7].

\section{Risk-Adapted Treatment}

Observation alone is reasonable for MIPSS70+ version 2.0 "low" and "very low" risk disease (estimated 10-year survival 65\%-92\%) [4] and intermediate-risk disease in the absence of treatment-requiring symptoms [7].

\section{Allogeneic Stem Cell Transplantation}

A preferred treatment option for MIPSS70+ version 2.0 "high" and "very high" risk disease (estimated 10-year survival $0-13 \%$ ) [4] since it can induce molecular remission and complete resolution of bone marrow fibrosis. Failure to achieve molecular remission on day 180 post allograft is associated with subsequent clinical relapse. Bone marrow fibrosis regresses rapidly after allogeneic SCT. About $60 \%$ of the patients have a complete or nearly complete remission of bone marrow fibrosis on day +100 and the percentage of patients increased to $90 \%$ at day+180 [8]. A careful selection of patients according to disease and transplantspecific risk factors is mandatory [8].

\section{Clinical Trial}

participation in a clinical trial might be the best approach in patients with intermediate risk disease (estimated 10-year survival 30\%) requiring treatment [4]. Imetelestat is a telomerase inhibitor that shows the most intriguing results among the drugs tested in MF. Response rates were 32\% among patients without an ASXL1 mutation. Complete response was 38\% among patients with a mutation in SF3B1 or U2AF1. Momelotinib (MMB, GS-0387, CYT387) is a JAK1 and JAK2 inhibitor undergoing phase-3 study. Anemia and spleen responses were $59 \%$ and $48 \%$, respectively. Most patients experienced constitutional symptoms improvement. Everolimus is an mTOR inhibitor in a phase $1 / 2$ study. More than $50 \%$ reduction in splenomegaly occurred in $20 \%$ of the evaluated patients and the constitutional symptoms response was $69 \%$. Drug effect on anemia was modest and on JAK2V617F burden was negligible [3].

\section{Management of Refractory Disease and Specific Disease Complications (Symptom-directed Therapy)}

Symptom-directed conventional drug therapy, radiotherapy, or splenectomy is advised in MIPSS70 + version 2.0 intermediaterisk patients [7] or higher risk patients that are not eligible for SCT or clinical trial participation [7]. These treatment options including use of JAK2 inhibitors [4] are mostly palliative and unlikely to modify the natural history of the disease or prolong survival [7]. They should not be used in the absence of clear treatment indication [4].
Anemia is best managed by erythropoiesis promoting drugs such as androgen preparations, danazol, thalidomide, and prednisone [7]. Pomalidomide (thalidomide analog) might alleviate anemia in a subset of JAK2-mutated patients without marked splenomegaly or excess circulating blasts [4]

i. Localized bone pain and symptomatic non-hepatosplenic EMH responds to involved-field radiotherapy [7].

ii. Up to $50 \%$ of patients with massive refractory splenomegaly respond to monthly courses of intravenous cladribine (2-chlorodeoxyadenosine). Severe reversible cytopenia being the main toxicity. Interferon-alfa (standard and pegylated) is not generally recommended since it causes minimal reduction in splenomegaly [1].

iii. Splenectomy is indicated in hydroxyurea-refractory splenomegaly, symptomatic portal hypertension, thrombocytopenia and frequent red blood cell transfusions [3]. Older age, leukocytosis, excess circulating blasts, and transfusion need are risk factors for inferior post-splenectomy survival [7]. Perioperative complications included infections, abdominal vein thrombosis and bleeding. Approximately $10 \%$ of patients experienced progressive hepatomegaly and $29 \%$ experienced thrombocytosis after splenectomy. Overall perioperative mortality rate was $9 \%$. Median survival after splenectomy was 19 months [3].

iv. Ruxolitinib (JAK inhibitor) is effective in alleviating constitutional symptoms and marked splenomegaly. Sooner or later, most patients become refractory to both hydroxyurea and ruxolitinib, and might require splenectomy [7].

\section{Practical Aspects of using Ruxolitinib}

\section{Ruxolitinib withdrawal Syndrome}

The mechanism of action of ruxolitinib is based on its nonspecific ability to suppress inflammatory cytokines. Long-term outcome disclosed a $92 \%$ treatment discontinuation rate after a median time of 9.2 months. Withdrawal symptoms occurring during ruxolitinib treatment discontinuation are characterized by acute relapse of disease symptoms, accelerated splenomegaly, worsening of cytopenias and occasional hemodynamic decompensation including a septic shock like syndrome [4].

\section{Why JAK Inhibitor therapy is not Disease modifying in Myelofibrosis despite their Potent in Vitro Inhibitory Activity?}

Absence of consistent molecular and/or pathologic responses in the tested JAK inhibitors in the clinic is proposed to be due to the frequency of mutations in non-canonical MPN-relevant genes (e.g. ASXL1, SRSF2), inadequate drug exposure and the highly variable JAK2V617F variant allele frequency (1-100\%), higher levels being seen in post-ET/PV MF than in PMF. The proposed heterodimeric JAK-STAT activation despite sustained JAK2 inhibition may play a role. JAK inhibitors may be more efficacious if used earlier in the proliferative/cellular phase, where, there may be favorable changes in BM architecture [5]. 


\section{Timing of SCT in Ruxolitinib Treated Patients}

Allogeneic SCT in PMF is currently associated with at least $50 \%$ rate of transplant-related deaths or severe morbidity (e.g. Graft vs. host disease) regardless of intensity of conditioning regimens used. There is much interest in evaluating the use of JAK inhibitors before Allogeneic SCT [4]. Transplanted patients after clinical response to JAKi showed improvement of their symptom burden, performance status and reduction of splenomegaly, thus facilitating more intensive therapeutic approaches, augmented hematological recovery and improved graft function compared with those who demonstrated loss of response or progressive disease [6]. In addition, ruxolitinib is hypothesized to modulate $\mathrm{T}$-, natural killer, and dendritic cell function and may alter the incidence and perhaps grade of graft-versus-host disease rates. Drawbacks of ruxolitinib therapy before SCT include drug-related cytopenia, rare risk of tumor lysis syndrome or unexpected toxicity. Longer follow-up is required to determine later outcomes [6].

\section{Selection of Salvage therapy after JAK Inhibitor Failure is Challenging}

Clinical (e.g., anemia/thrombocytopenia grade, spleen size), biological (e.g., poor-risk molecular or cytogenetic abnormalities), and patient-specific (e.g., age, performance status) factors, and the reason(s) for JAK inhibitor failure (e.g., inadequate dosing due to drug-related toxicity) has to be considered in JAK inhibitor therapy failure. Symptom based approach is frequently necessary in SCT-ineligible patients and absence of an identifiable treatment target. An agent with a novel mode of action is preferred If JAK inhibitor failure relates to dose-limiting toxicity. Addition of

DOI: 10.19080/CTOIJ.2018.12.555847 interferon- $\alpha$ or hydroxyurea to the JAK inhibitor may restore clinical response although safety data for such combinations is lacking. In other instances, participation in a clinical trial with an alternative JAK inhibitor may be appropriate [5].

\section{Conclusion}

Although the therapeutic algorithms for MF have recently altered, still more researches are needed to find a surrogate marker for disease response and to fill the gap between molecular profile of the patients and their application in clinical practice.

\section{References}

1. Iurlo A, Cattaneo D (2017) Treatment of myelofibrosis: old and new strategies. Clinical Medicine Insights: Blood Disorders 10: 1-10.

2. Guglielmelli P, Lasho TL, Rotunno G, Mudireddy M, Mannarelli C, et al. (2017) MIPSS70: Mutation-Enhanced International Prognostic Score System for transplantation-age patients with primary myelofibrosis. J Clin Oncol 36(4): 310-318.

3. Tefferi A (2016) Primary myelofibrosis: 2017 update on diagnosis, risk-stratification and management. Am J Hematol 91(12): 1261-1271.

4. Tefferi A (2018) Primary myelofibrosis: 2019 update on diagnosis, risk-stratification and management. Am J Hematol 93(12): 1551-1561.

5. Pardanani A, Tefferi A (2018) How we treat myelofibrosis after failure of JAK inhibitors. Blood 132(5): 492-500.

6. Harrison CN, McLornan DP (2017) Current treatment algorithm for the management of patients with myelofibrosis, JAK inhibitors, and beyond. Hematology Am Soc Hematol Educ Program 2017(1): 489497.

7. Tefferi A, Guglielmelli P, Pardanani A, Vannucchi AM (2018) Myelofibrosis treatment algorithm 2018. Blood Cancer J 8(8): 72.

8. Kröger N (2018) Stem cell transplantation in myelofibrosis. Educational Updates in Hematology Book 2(S2): 146-148.

\section{Your next submission with Juniper Publishers} will reach you the below assets

- Quality Editorial service

- Swift Peer Review

- Reprints availability

- E-prints Service

- Manuscript Podcast for convenient understanding

- Global attainment for your research

- Manuscript accessibility in different formats

( Pdf, E-pub, Full Text, Audio)

- Unceasing customer service

Track the below URL for one-step submission https://juniperpublishers.com/online-submission.php 